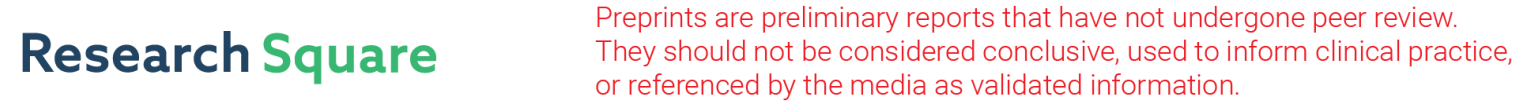 \\ Theoretical Basis of Face Contact Pressure Design of Zero-leakage Mechanical Seal
}

\section{Wei Zheng}

Nanjing Forestry University https://orcid.org/0000-0002-2067-1392

Jianjun Sun ( $\nabla$ sunjianjun@njfu.edu.cn )

Nanjing Forestry University https://orcid.org/0000-0001-9456-9334

Chenbo Ma

Nanjing Forestry University

Qiuping Yu

Nanjing Forestry University

\section{Research Article}

Keywords: Mechanical seal, Contact pressure, Leakage rate, Wear rate, Frictional heat, Percolation theory

Posted Date: December 10th, 2021

DOI: https://doi.org/10.21203/rs.3.rs-1118674/v1

License: (9) This work is licensed under a Creative Commons Attribution 4.0 International License.

Read Full License 


\title{
Theoretical basis of face contact pressure design of zero-leakage mechanical seal
}

\section{Zheng Wei}

College of Mechanical and Electrical Engineering, Nanjing Forestry University, Nanjing, Jiangsu, PR China, 210037, e-mail: zhengwei@njfu.edu.cn

\section{Sun Jianjun ${ }^{1}$}

College of Mechanical and Electrical Engineering, Nanjing Forestry University, Nanjing, Jiangsu, PR China, 210037, e-mail: sunjianjun@njfu.edu.cn

\section{Ma Chenbo}

College of Mechanical and Electrical Engineering, Nanjing Forestry University, Nanjing, Jiangsu, PR China, 210037, e-mail: machenbo@njfu.edu.cn

\section{Yu Qiuping}

College of Mechanical and Electrical Engineering, Nanjing Forestry University, Nanjing Jiangsu, PR China, 210037, e-mail: yuqiuping03@njfu.edu.cn

\begin{abstract}
Based on the percolation theory, the critical porosity of zero-leakage at the wetting and non-wetting sealing interface working in liquid medium is first discussed. The influence of end-face frictional heat on end-face friction and wear is then investigated. The design criteria for the face contact pressure of mechanical seals with zero-leakage and long-life operation are established. Afterwards, the face contact pressure range of the mechanical seal working in conventional different liquid medium is calculated, and the influence of different working conditions speed, medium temperature and pressure on the face contact pressure range change is analyzed. Existing studies have shown that mechanical seals can achieve zero-leakage and long-life operation. As for the wettable sealing interface, the minimum face contact pressure, corresponding to the zero-leakage condition, is only related to the morphological parameters of the sealing interface, and has nothing to do with the sealing medium. Under the rotating and stationary rings physical parameters and given working conditions, the face contact pressure range of the sealing medium water and propane propylene is $0.477 \sim 1.132 \mathrm{MPa}$. The diesel sealing medium has a larger face contact pressure range than that of water and propane

1 Corresponding author
\end{abstract}


propylene, which can reach 0.477 2.183 MPa. The working condition speed, medium temperature and medium pressure have an influence on the face contact pressure range, while the influence of the working condition speed is the most significant.

Keywords: Mechanical seal; Contact pressure; Leakage rate; Wear rate; Frictional heat; Percolation theory

\section{Introduction}

Mechanical seal is the main shaft seal form of rotating equipment such as centrifugal compressors, centrifugal pumps, steam turbines, etc. Its performance parameters include the leakage rate and wear life [1]. Besides the working condition parameters, the mechanical parameter of the face contact pressure is the most critical factor affecting the mechanical seal performance [2]. Increasing the face contact pressure is beneficial in reducing the void ratio between the sealing interface, and achieves zero leakage. However, excessive face contact pressure will increase the wear of the sealing interface and shorten the service life of the mechanical seal $[3,4]$. For this reason, many researchers have conducted in-depth explorations on the selection principle of the face contact pressure.

For instance, Mayer [5] defined the face contact pressure as the resultant force of the axial force acting on the unit sealing surface area, including the liquid film force and the asperity bearing capacity. He mentioned that the leakage rate is inversely proportional to the square of the face contact pressure, and the amount of wear increases with the increase of the contact pressure. Lebeck [6] and $\mathrm{Gu}$ Yongquan [7, 8] clarified from a microscopic point of view that the face contact pressure is only the contact bearing capacity of the asperity per unit nominal sealing surface area, which is equal to the product of the compressive strength of a relatively weak material and the ratio of the bearing area of the asperity. Salant et al. [9] used the plastic contact model, by assuming that the asperity height on the end face is Gaussian. They determined the contact pressure at any radius on the contact face. Based on the GW model, Elhanafi et al. [10] developed a contact load expression for a flat surface and a rough surface with Gaussian distribution of asperity height. Sun Jianjun et al. [11] and Li Xiaopeng et al. [12] used fractal parameters that are independent of the measurement scale, in order to describe the cross-sectional 
profile of the microchannel. They then revealed the law of microchannel interface size, changing with contact pressure. These studies have contributed to the safe operation of mechanical seals. However, gaps or microchannels at the sealing interface under operable contact pressure still exist. It is impossible to explain the zero-leakage and long period operation of the mechanical seals, such as the main pump and the refrigeration compressor of the sodium-cooled fast reactor, for example. In fact, the sealing interface is composed of the rotating and stationary rings surfaces obtained by machining. In addition, although voids exist, the size of the void ratio can be controlled by applying contact pressure, ensuring that there is no percolation at the sealed interface and achieving zero-leakage [13]. Therefore, determining the best face contact pressure range is the key to achieving zero-leakage and long-life mechanical seal operation.

This paper intends to start from the zero-leakage of the sealing interface, based on the percolation theory, to discuss the critical porosity of the wetting and non-wetting sealing interface in liquid media. The temperature rise of the sealing interface caused by frictional heat and the resulting deep wear rate are investigated. The requirements of zero-leakage rate and wear rate not exceeding the standard are combined to determine the range of the seal face contact pressure, and establish the theoretical basis for the design of the seal face contact pressure. Consequently, the face contact pressure range of the conventional sealing medium, clean water, diesel and propane propylene, is calculated and analyzed.

\section{The critical porosity at zero leakage}

In the initial state or wear process of the sealing interface composed of rotating and stationary rings, there are always micro-convex contacts and voids. The sealing interface are uneven under the microscope, as shown in Fig. 1 [4]. When the porosity of the sealing interface reaches the percolation threshold, microchannels penetrating the sealing surface at the sealing interface will appear [14]. On the contrary, microchannels in the non-percolated sealed interface with a porosity less than the percolation threshold do not exist. However, whether there should be fluid flow at the sealing interface in the percolation state depends on whether the driving force on both microchannel sides is greater than the resistance of the fluid to flow [15]. 


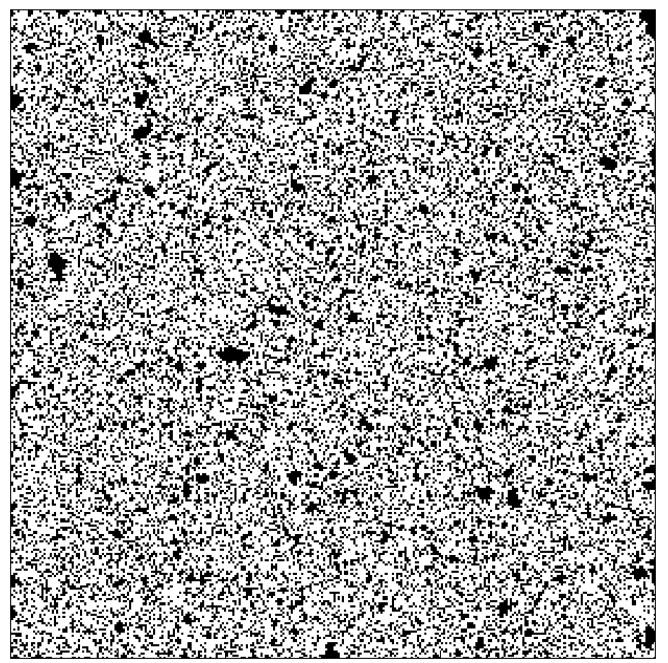

Fig. 1. Surface topography of a mechanical seal ring under microscope

The wettability of the sealed medium to the rotating and stationary rings materials is different. The effect of the capillary force formed in the microchannel on the sealing interface, is also different.

For the sealing interface composed of rotating and stationary rings materials that cannot be wetted, the capillary force formed by the sealing medium in the microchannel prevents the leakage of the sealed medium [4]. In order to ensure that the sealed medium exists in the sealed interface to achieve lubrication, while not leaking from the microchannel, it is necessary to control the size of the microchannel pore. Increasing the face contact pressure can reduce the void ratio of the sealing interface, reduce the radius of the void capillary, increase the capillary force and improve the leakage resistance of the sealing interface [3], as shown in Fig. 2.

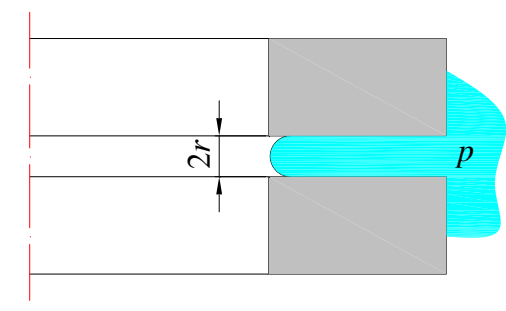

a) Large diameter microchannel

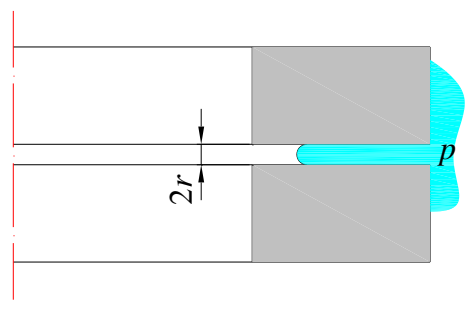

b ) Small diameter microchannel

Fig. 2. Not infiltrated capillary force formed in the microchannel

The relationship between the capillary force of the microchannel at the sealed interface and 
the radius of the microchannel $(r)$ can be expressed by the interface Laplace equation [16]:

$$
p=\frac{2 \zeta \cos \varphi}{r}
$$

where $p$ is the capillary force, $\zeta$ represents the surface tension of the liquid, $\varphi$ denotes the contact angle, and $r$ is the radius of the microchannel.

When $p$ is the medium pressure, $r_{\mathrm{c}}$ is the critical radius of the zero-leakage microchannel. The porosity maintained by the sealed interface of the microchannel with a critical radius is the critical porosity, in the case of zero-leakage. In order to ensure zero-leakage, it is necessary to guarantee that the pore size of the microchannel at the sealing interface is smaller than the critical radius $\left(r_{\mathrm{c}}\right)$ of the zero-leakage microchannel.

For the rotating and stationary rings face materials that can be infiltrated by the sealing medium, there should be no microchannels at the sealing interface, because the capillary force will push the sealed medium to leak. In order to ensure zero-leakage, it is necessary to control the void ratio of the sealing interface to be less than the percolation threshold of 0.312 [14].

\section{Theoretical analysis of wear rate of sealing interface}

Increasing the face contact pressure improves the sealing performance of the sealing interface. It also increases the friction of the sealing interface [17]. The generation of frictional heat and the increase of the end surface temperature degrade the performance of the rotating and stationary rings materials, and bring the risk of excessive wear rate to the mechanical seal [18].

\subsection{Calculation of the sealing interface frictional heat}

Under the action of end face load, the thermal strength $(\dot{Q})$ of the sealing interface due to friction is expressed as [6]:

$$
\dot{Q}=\pi\left(r_{\mathrm{o}}^{2}-r_{\mathrm{i}}^{2}\right) p_{\mathrm{g}} f U
$$

where $r_{\mathrm{i}}$ and $r_{\mathrm{o}}$ are respectively the inner and outer radius of the sealing surface, $p_{\mathrm{g}}$ represents the specific load of the end face such that $p_{\mathrm{g}}=p_{\mathrm{c}}+p_{\mathrm{m}}, p_{\mathrm{c}}$ denotes the face contact pressure, $p_{\mathrm{m}}$ is the 
fluid film pressure on the end face, $U$ is the linear velocity of the middle diameter of the sealing surface, and $f$ is the total friction coefficient of the sealing interface given by:

$$
\begin{gathered}
f=\frac{F_{\mathrm{mf}}+F_{\mathrm{cf}}}{W} \\
F_{\mathrm{mf}}=\frac{2}{r_{\mathrm{i}}+\mathrm{r}_{\mathrm{o}}} \int_{0}^{\beta} \int_{r_{i}}^{r_{o}}\left(\frac{\mu r \omega}{h}-\frac{h}{2} \frac{\partial p}{r \partial \theta}\right) r^{2} \mathrm{~d} r \mathrm{~d} \theta \\
F_{\mathrm{cf}}=\frac{2}{r_{\mathrm{i}}+\mathrm{r}_{\mathrm{o}}} \int_{0}^{2 \pi-\beta} \int_{r_{i}}^{r_{o}} p_{\mathrm{c}} f_{c} r^{2} \mathrm{~d} r \mathrm{~d} \theta
\end{gathered}
$$

where $W$ is the end face load such that $W=p_{\mathrm{g}} A_{\mathrm{a}}, A_{\mathrm{a}}$ represents the nominal contact area of sealing surface, $\beta=2 \pi\left(h \phi_{0}-\delta\right) /(h-\delta), h$ denotes the fluid film thickness in the non-contact zone of the sealing interface, $\delta$ is the compression of the asperity under the action of $p_{\mathrm{c}}, \mu$ is the fluid viscosity, and $f_{\mathrm{c}}$ is the adhesive friction factor.

When the sealing interface is in an elastic contact state, the adhesive friction factor $\left(f_{\mathrm{c}}\right)$ can be computed as:

$$
f_{\mathrm{c}}=f_{\mathrm{e}} \approx \alpha \frac{\tau_{\mathrm{c}}}{E^{*}\left(\sigma / \beta^{*}\right)}
$$

where $\alpha$ is the component of the asperities contact area which can be determined as in [19], $\tau_{\mathrm{c}}$ represents the average value of the dry friction shear strength, $E^{*}$ denotes the composite elastic modulus of the rotating and stationary rings, $\sigma$ is the equivalent surface mean square error of asperities [6] such that $\sigma=\sqrt{\sigma_{1}^{2}+\sigma_{2}^{2}}$, and $\beta^{*}$ is the composite correlation length of the asperity [20] generally equal to $0.8 \mathrm{~mm}[21]$.

When the sealing interface is in a plastic contact state, the adhesion friction factor $\left(f_{\mathrm{c}}\right)$ can be calculated as:

$$
f_{\mathrm{c}}=f_{\mathrm{p}}=\frac{\tau_{\mathrm{c}}}{H_{\mathrm{s}}}
$$

where $H_{\mathrm{s}}$ is the material hardness of the softer ring in the sealing interface matched ring. 
When the sealing interface is in an elastic-plastic contact state, the adhesion friction factor $\left(f_{c}\right)$ can be computed as:

$$
f_{\mathrm{c}}=f_{\mathrm{ep}}=f_{\mathrm{e}}+\left(f_{\mathrm{p}}-f_{\mathrm{e}}\right) f(a)
$$

where $f(a)$ is the template function given by [22]:

$$
f(a)=-2\left(\frac{a-a_{\mathrm{ec}}}{a_{\mathrm{pc}}-a_{\mathrm{ec}}}\right)^{3}+3\left(\frac{a-a_{\mathrm{ec}}}{a_{\mathrm{pc}}-a_{\mathrm{ec}}}\right)^{2}
$$

where $a_{\mathrm{ec}}$ and $a_{\mathrm{pc}}$ are the critical area of the asperity elastic deformation and plastic deformation, respectively.

\subsection{Calculation of the sealing interface temperature}

The frictional heat generated by the friction of the sealing interface, satisfies the classical equation of isotropic solids heat conduction [23]:

$$
\frac{\partial^{2} T}{\partial r^{2}}+\frac{1}{r} \frac{\partial T}{\partial r}+\frac{1}{r^{2}} \frac{\partial^{2} T}{\partial^{2} \theta}+\frac{\partial^{2} T}{\partial z^{2}}+\frac{Q / V}{K}=\frac{c_{\mathrm{p}} \rho}{K} \frac{\partial T}{\partial t}
$$

where $T$ is the temperature rise, $K$ represents the thermal conductivity, $\rho$ denotes the density, $c_{\mathrm{p}}$ is the specific heat capacity at a constant pressure, $t$ represents the time, $Q / V$ denotes the internal heat generation, and $V$ is the volume.

Assuming there is no heat exchange between the rotating and stationary rings and the outside world, and the frictional heat is distributed between the two rings as $\chi_{\mathrm{h}}:\left(1-\chi_{\mathrm{h}}\right)$, the sealing interface with different contact stresses will obtain different temperature rises [24].

Under the action of high contact stress, that is, when the specific load $\left(p_{\mathrm{g}}\right)$ of the end face is close to the hardness $\left(H_{\mathrm{s}}\right)$ of the weaker material or $A_{\mathrm{r}} / A_{\mathrm{a}} \approx 1$, the contact and friction heat of the rotating and stationary rings relative to high-speed sliding (Pelect number $L=U l / \kappa>10$ ) and low-speed sliding $(\mathrm{L}<0.5)$ are uniform. The temperature rise of the end face is given by $[21,24]$ :

When sliding at high-speed $(U l / \kappa>10)$ : 


$$
\theta_{\max }=1.6 \frac{\chi_{\mathrm{h}} q}{\rho_{1} c_{p 1} U}\left(\frac{U l}{\kappa_{1}}\right)^{1 / 2}
$$

where $\chi_{\mathrm{h}}=\left[1+\left(\frac{K_{2}}{K_{1}}\right)\left(\frac{\kappa_{1}}{U l}\right)\right]^{-1}$, and $\kappa$ is the thermal diffusivity equal to $\frac{K}{\rho c_{\mathrm{p}}}$.

When sliding at low-speed $(U l / \kappa<0.5)$ :

$$
\theta_{\max }=0.64 \frac{\chi_{\mathrm{h}} q l}{K_{1}}\left[\operatorname{arsinh}\left(\frac{b}{l}\right)+\left(\frac{b}{l}\right) \operatorname{arsinh}\left(\frac{l}{b}\right)\right]
$$

where $q$ is the heat flux density equal to $p_{\mathrm{g}} f U, l$ is the half width of the rectangular heat source in the $\mathrm{x}$ direction (equal to $\pi\left(r_{\mathrm{i}}+r_{\mathrm{o}}\right) / 2$ for mechanical seal rings), and $b$ is the half width of the sealing surface.

The contact and frictional heat formed on the sealing interface are not uniform in the relative sliding of the rotating and stationary rings under low contact stress $\left(A_{\mathrm{r}} / A_{\mathrm{a}}<<1\right)$ [25]. The rotating and stationary rings sealing interfaces, formed by the matching of different surface roughnesses, have different temperature rises during relative sliding.

(1) Calculation of the sliding temperature rise with roughly equivalent surface roughness

When sliding at high-speed $\left(3 U d_{\max } / 16 \kappa>10\right)$ :

$$
\theta_{\max }=\chi_{\mathrm{L}}\left[\frac{0.95 f p_{\mathrm{g}}\left(\frac{A_{\mathrm{a}}}{A_{\mathrm{r}}}\right)\left(\frac{U d_{\max }}{\kappa_{1}}\right)^{1 / 2}}{\rho_{1} c_{\mathrm{p} 1}}+\frac{1.5 f p_{\mathrm{g}}\left(\frac{U l}{\kappa_{1}}\right)^{1 / 2}}{\rho_{1} c_{\mathrm{p} 1}}\right]
$$

When sliding at low-speed $(U l / \kappa<0.5)$ :

$$
\theta_{\max }=\left[\frac{f p_{\mathrm{g}} U}{K_{1}+K_{2}}\right]\left[0.33\left(\frac{A_{\mathrm{a}}}{A_{\mathrm{r}}}\right) d_{\max }+\psi l\right]
$$

where $\chi_{\mathrm{L}}=\left[1+\left(\frac{K_{2} \rho_{2} c_{\mathrm{p} 2}}{K_{1} \rho_{1} c_{\mathrm{p} 1}}\right)^{1 / 2}\right]^{-1}, d_{\max }$ is the maximum contact diameter of the asperities, and $\psi$ is 
the area coefficient of the heat source equal to 1.12 [24].

(2) Calculation of sliding temperature rise between rough surface and flat surface

When sliding at high-speed $(U \bar{d} / 2 \kappa>10)$ :

$$
\theta_{\max }=\chi_{\mathrm{L}}\left[\frac{1.5 f p_{\mathrm{g}}\left(\frac{A_{\mathrm{a}}}{A_{\mathrm{r}}}\right)\left(\frac{U \bar{d}}{2 \kappa_{1}}\right)^{1 / 2}}{\rho_{1} c_{\mathrm{p} 1}}+\frac{1.5 f p_{\mathrm{g}}\left(\frac{U l}{\kappa_{1}}\right)^{1 / 2}}{\rho_{1} c_{\mathrm{p} 1}}\right]
$$

When sliding at low-speed $(U l / \kappa<0.5)$ :

$$
\theta_{\max }=\left[\frac{f p_{\mathrm{g}} U}{K_{1}+K_{2}}\right]\left[0.5 \psi\left(\frac{A_{\mathrm{a}}}{A_{\mathrm{r}}}\right) \bar{d}+\psi l\right]
$$

where $\bar{d}$ is the average diameter of the asperity.

$A_{\text {am }} / n(a) a$ can be used to obtain the contact surface area of a single asperity. $a=\frac{\pi}{4} \bar{d}^{2}$ is then used to find $\bar{d}$, where $A_{\text {am }}$ is the calculated nominal area given by:

$$
A_{\mathrm{am}}=\frac{D}{2-D} \psi^{(2-D) / 2} \frac{\pi}{4} d^{2}
$$

where $d$ is the bottom diameter of the largest asperity and $D$ denotes the fractal dimension of the sealing end surface.

Note that $n(a) a$ represents the number of asperities, which can be expressed as:

$$
n(a) a=\frac{D}{2} \psi^{(2-D) / 2}\left(\frac{\pi}{4} d^{2}\right)^{D / 2} a^{-D / 2}
$$

\subsection{Calculation of the wear rate of the sealing interface}

According to the obtained sealing interface temperature, the deformation state of the asperity on the soft ring is judged. The corresponding deep wear rate calculation formula can then be selected to calculate the depth wear rate. 
For plastic contact, the wear rate is given by [26]:

$$
\dot{d}=\dot{d}_{\mathrm{p}}=\frac{k p_{\mathrm{g}} U}{H_{\mathrm{s}}}
$$

where $k$ is the wear coefficient.

For elastic contact, the wear rate is expressed as [24]:

$$
\dot{d}=\dot{d}_{\mathrm{e}}=\frac{k p_{\mathrm{g}} U}{E^{*}\left(\sigma / \beta^{*}\right)}
$$

For elastic-plastic contact, the wear rate is given by:

$$
\dot{d}=\dot{d}_{\mathrm{ep}}=\dot{d}_{\mathrm{e}}+\left(\dot{d}_{\mathrm{p}}-\dot{d}_{\mathrm{e}}\right) f(a)
$$

\section{Design criteria for face contact pressure}

\subsection{Design criteria}

In order to achieve the zero-leakage and long-life operation of the mechanical seal, the microchannel radius of the sealing interface should be smaller than the critical radius of the zero-leakage microchannel, or no microchannel should exist. Simultaneously, it should meet the wear rate requirement $(\dot{d}<0.02 \mathrm{~mm} / 100 \mathrm{~h})[27]$.

The radius of the microchannel is smaller than the critical radius of the zero-leakage microchannel, which is achieved by controlling the seal face contact pressure. The change of the face contact pressure affects the porosity of the sealing interface, the formation of the microchannel and its radius scale [28]. In addition, the face contact pressure increases, the void ratio of the sealing interface decreases, the radius of the microchannel decreases, and no microchannel exists [14]. We then continue to increase the face contact pressure to compact the sealing interface so that the porosity becomes zero. However, the porosity decrease will reduce the fluid film of the sealing interface, increase the frictional heat, decrease the material performance, and increase the wear rate [29]. When the wear rate is greater than $0.02 \mathrm{~mm} / 100 \mathrm{~h}$, the wear of the sealing interface exceeds the standard. For this reason, it is necessary to determine the face contact 
pressure range to ensure the zero-leakage and long-life operation of the mechanical seal.

Therefore, the design criteria for the face contact pressure of the mechanical seal can be given by:

(1) The wettable sealing interface:

$$
\left\{\begin{array}{l}
\phi=\frac{h \phi_{0}-\delta}{h-\delta}=G\left(p_{\mathrm{c}}\right)<0.312 \\
\dot{d}=H\left(p_{\mathrm{c}}\right)<0.02 \\
\delta=G^{D-1} l^{2-D}\left(1-\cos \frac{\pi \sqrt{a}}{2 d}\right)
\end{array}\right.
$$

(2) The non-wettable sealing interface:

When the sealing interface is in a non-permeable state $(\phi<0.312)$, the design criterion for the face contact pressure is computed as in Eq. (22).

When the sealing interface is in a state of percolation $(\phi>0.312)$, the design criterion for the face contact pressure is given by:

$$
\left\{\begin{array}{l}
\phi=\frac{h \phi_{0}-\delta}{h-\delta}=G\left(p_{\mathrm{c}}\right)>0.312 \\
r=\frac{h-\delta}{2}=S\left(p_{\mathrm{c}}\right)<r_{\mathrm{c}} \\
\dot{d}=H\left(p_{\mathrm{c}}\right)<0.02 \\
r_{\mathrm{c}}=\frac{2 \zeta \cos \varphi}{p_{\mathrm{s}}} \\
\delta=G^{D-1} l^{2-D}\left(1-\cos \frac{\pi \sqrt{a}}{2 d}\right)
\end{array}\right.
$$

where $\phi_{0}$ is the initial porosity of the sealing interface, $\phi$ represents the porosity after the sealing interface is deformed under load, $p_{\mathrm{s}}$ denotes the medium pressure, $G\left(p_{\mathrm{c}}\right), S\left(p_{\mathrm{c}}\right)$ and $H\left(p_{\mathrm{c}}\right)$ are the defined characterization functions that are used to characterize the complex relationship between the face contact pressure $\left(p_{c}\right)$, porosity $(\phi)$, microchannel radius $(r)$ and wear rate $(\dot{d})$.

Note that $G\left(p_{\mathrm{c}}\right)$ and $S\left(p_{\mathrm{c}}\right)$ can be computed as in [19].

(1) Characterization function $G\left(p_{c}\right)$ 
With the continuous increase of the face contact pressure $\left(p_{\mathrm{c}}\right)$, the contact state of the sealing interface asperities will undergo three stages of elastic deformation, elastic-plastic deformation and plastic deformation. There are different functional relationships between the face contact pressure $\left(p_{\mathrm{c}}\right)$ and the contact area $(a)$ of the asperities under different contact states. The corresponding compression amount $(\delta)$ can be determined in the end. Therefore, the characterization function $G\left(p_{\mathrm{c}}\right)$ depends on the deformation of the asperity. The face contact pressure has a direct effect on the deformation of the asperity.

\section{(2) Characterization function $S\left(p_{\mathrm{c}}\right)$}

Similar to $G\left(p_{\mathrm{c}}\right)$, there will be different functional relationships between the face contact pressure $\left(p_{\mathrm{c}}\right)$ and the contact area $(a)$ with the asperity under different contact states, as $p_{\mathrm{c}}$ increases. Finally, the corresponding compression amount $(\delta)$ can be determined, in order to obtain the microchannel radius $(r)$. Therefore, in different contact states, the characteristic function $S\left(p_{\mathrm{c}}\right)$ of the face contact pressure $\left(p_{\mathrm{c}}\right)$ and the microchannel radius $(r)$, will also be different

\section{(3) Characterization function $H\left(p_{\mathrm{c}}\right)$}

The functional relationship between the face contact pressure $\left(p_{\mathrm{c}}\right)$ and the wear rate $(\dot{d})$ in different contact states, can be determined by Eq. (19), (20) and (21). Therefore, function $H\left(p_{\mathrm{c}}\right)$ under different contact states, can be computed as in Eq. (19), (20) and (21)

Note that the design criterion (cf. Eq. (22)) for the face contact pressure of the wettable sealing interface is in the non-percolation state $(\phi<0.312)$. However, in the percolation state $(\phi>$ 0.312), there is a leakage channel in the wettable seal interface, but a mechanical seal zero-leakage can still be achieved, which is not discussed in this paper. The zero-leakage mechanism under percolation state $(\phi>0.312)$ is related to the interaction between the fluid intermolecular force, fluid dynamic and static pressure mechanism, and fluid shear action. The zero-leakage mechanism under this state and its face contact pressure design criterion, should be further studied in future work. 


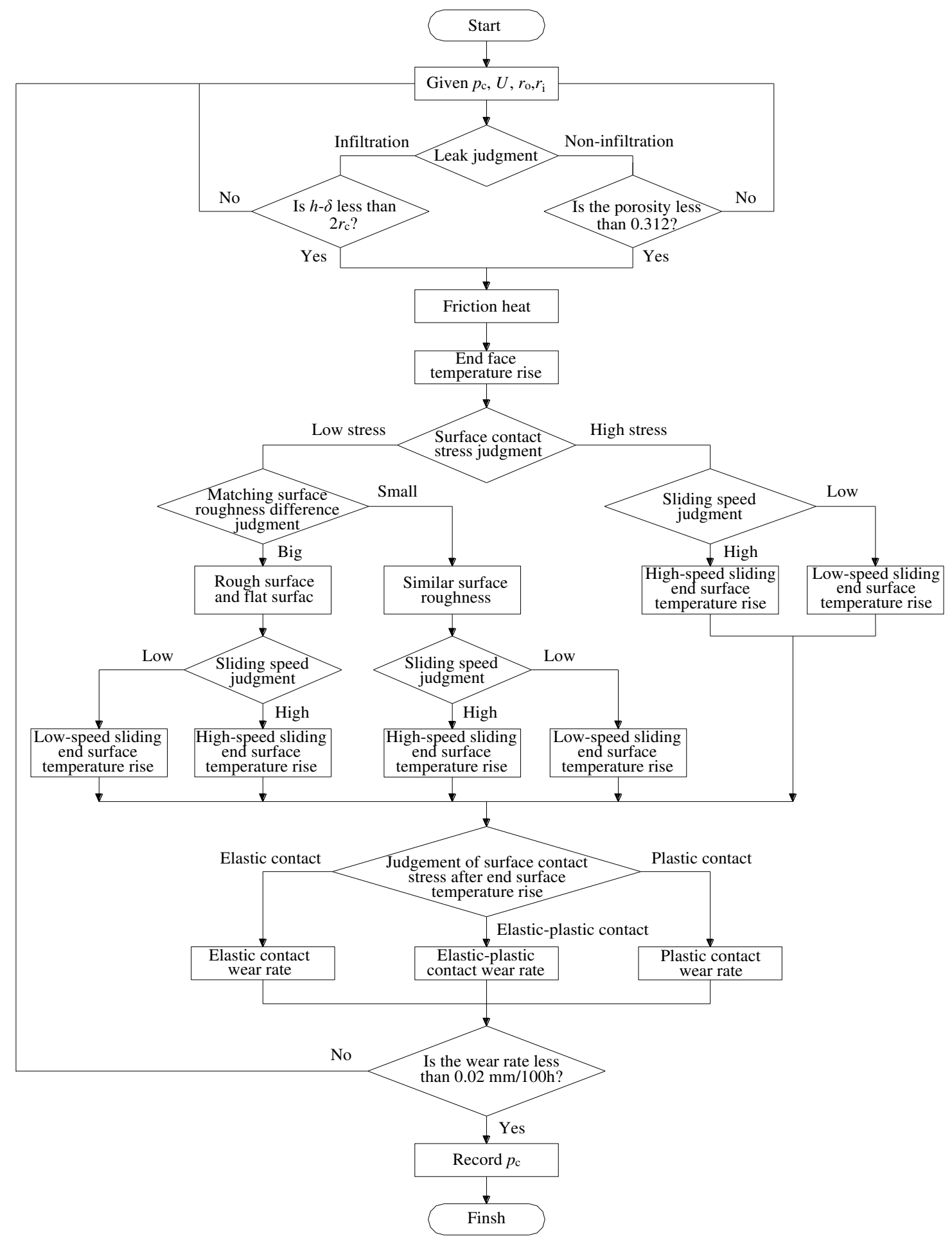

Fig. 3. Solution process of the face contact pressure range

\subsection{Calculation process}

Eq. (22) and Eq. (23) present the design criteria for the face contact pressure of mechanical seals, with two different wetted surfaces under the permissible wear rate $(\dot{d}<0.02 \mathrm{~mm} / 100 \mathrm{~h})$, in the case of zero-leakage. It is important to mention that the porosity $(\phi)$ and microchannel radius 
$(r)$ are negatively correlated with $p_{\mathrm{c}}$, while the wear rate $(\dot{d})$ is positively correlated with $p_{\mathrm{c}}$. Therefore, the design criterion in Eq. (22) or Eq. (23) can be used to determine the face contact pressure range of the mechanical seal, during zero-leakage operation under the allowable wear rate $\left(p_{\mathrm{cmin}} \sim p_{\mathrm{cmax}}\right)$.

Fig. 3 shows the specific solution flow of the face contact pressure range, under the zero-leakage and long-life operating state developed in this paper.

\section{Calculation example}

The rotating ring $(\mathrm{SiC})$ and stationary ring (M106K), commonly used in mechanical seals, are selected. Based on the previously developed theoretical basis, the wettable sealing interface formed by several commonly used liquid mediums is investigated in the wear rate of $\dot{d}<0.02$ $\mathrm{mm} / 100 \mathrm{~h}$, and a value range of the face contact pressure of different sealing mediums in zero-leakage operation. The change of the face contact pressure range under different working condition speed, medium temperature and medium pressure, is also analyzed.

\subsection{The face contact pressure range of the conventional sealing medium}

Table 1 shows the physical property parameters of the sealing medium clean water, diesel oil and propane propylene, at $37{ }^{\circ} \mathrm{C}$ with a medium pressure of $1.275 \mathrm{MPa}$ [1]. Table 2 shows the structural parameters of the rotating and stationary rings, and their related material performance parameters $[1,5,30]$.

Table 1. Physical parameters of several commonly used sealing mediums

\begin{tabular}{ccccccc}
\hline Media type & $\begin{array}{c}\text { Temperature } \\
T /{ }^{\circ} \mathrm{C}\end{array}$ & $\begin{array}{c}\text { Medium } \\
\text { pressure } \\
p_{\mathrm{s}} / \mathrm{MPa}\end{array}$ & $\begin{array}{c}\text { Kinematic } \\
\text { viscosity } \\
\gamma / \mathrm{pa} \cdot \mathrm{s}\end{array}$ & $\begin{array}{c}\text { Density } \\
\rho /\left(\mathrm{kg} \cdot \mathrm{m}^{-3}\right)\end{array}$ & $\begin{array}{c}\text { Specific heat } \\
\text { capacity } \\
c_{\mathrm{p}} /\left(\mathrm{J} \cdot(\mathrm{kg} \cdot \mathrm{K})^{-1}\right)\end{array}$ & $\begin{array}{c}\text { Thermal } \\
\text { conductivity } \\
\mathrm{K} /\left(\mathrm{W} \cdot(\mathrm{m} \cdot \mathrm{K})^{-1}\right)\end{array}$ \\
\hline $\begin{array}{c}\text { Clean } \\
\text { water }\end{array}$ & 37 & 1.275 & $680 \times 10^{-6}$ & 993 & 4179 & 0.630 \\
$\begin{array}{c}\text { Diesel fuel } \\
\text { Propane }\end{array}$ & 37 & 1.275 & $4.55 \times 10^{-3}$ & 817 & 2130 & 0.218 \\
Propylene & 37 & 1.275 & $93 \times 10^{-6}$ & 473 & 2890 & 0.086 \\
\hline
\end{tabular}


Table 2. Structural parameters and material performance parameters of the rotating and stationary

\begin{tabular}{ccc}
\multicolumn{3}{c}{ rings } \\
\hline Attribute parameter & Hard ring & Soft ring \\
\hline Material & SiC & M106K \\
Elastic Modulus $E / \mathrm{GPa}$ & 480 & 26 \\
Poisson's ratio $\mu$ & 0.15 & 0.25 \\
Hardness H/MPa & $2.2 \times 10^{4}$ & 30 \\
End fractal dimension $D$ & 1.65 & 1.57 \\
End face scale factor $G / \mathrm{m}$ & $1 \times 10^{-9}$ & $5 \times 10^{-9}$ \\
Maximum height of surface & 0.28 & 2.13 \\
profile $R_{\mathrm{y}}$ & 62 & 68 \\
Inside diameter $d_{\mathrm{i}} / \mathrm{mm}$ & 82 & 79 \\
Outer diameter $d_{\mathrm{o}} / \mathrm{mm}$ & 3070 & 2400 \\
Density $\rho / \mathrm{kg} \cdot \mathrm{m}^{-3}$ & 670 & 750 \\
Specific heat capacity & & \\
$c_{\mathrm{p}} / \mathrm{J} \cdot(\mathrm{kg} \cdot \mathrm{K})^{-1}$ & 130 & 10 \\
Thermal Conductivity & & \\
$K / \mathrm{J} \cdot(\mathrm{m} \cdot \mathrm{K})^{-1}$ & 1000 & 220 \\
$T /{ }^{\circ} \mathrm{C}$ & 34 & 12.0 \\
Maximum $\mathrm{use}$ temperature & $2.1 \times 10^{4}$ &
\end{tabular}

It is important to mention that, as long as one surface can be wetted, the capillary force of the microchannel at the sealing interface will become the driving force for leakage. The sealing interface composed of the selected sealing medium and the rotating and stationary rings, is a surface that can be wetted. Therefore, to ensure that there is no leakage at the sealing interface, there should be no microchannels at the sealing interface. That is, the porosity of the sealing interface should be controlled to be less than the percolation threshold of 0.312 .

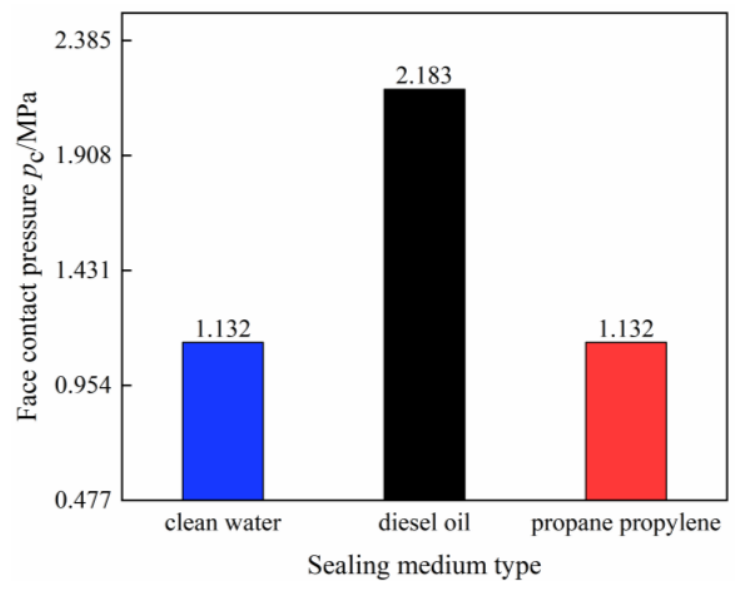

Fig. 4. The face contact pressure range of different sealing media

The initial operating speed and the medium temperature are respectively set to $3000 \mathrm{r} / \mathrm{min}$, and $37^{\circ} \mathrm{C}$, while the data of Table 1 and Table 2 are used for the other relevant parameters. The 
face contact pressure range of the sealing medium clean water, diesel oil and propane propylene under the allowable wear rate $(\dot{d}<0.02 \mathrm{~mm} / 100 \mathrm{~h})$ and the sealing interface zero-leakage operation, are obtained by calculation. The calculation results are shown in Fig. 4.

It can be seen from Fig. 4 that the face contact pressure range of clean water and propane propylene is $0.477 \sim 1.132 \mathrm{MPa}$. The face contact pressure range of diesel oil is $0.477 \sim 2.183 \mathrm{MPa}$, which is greater than that of clean water and propane propylene. In other words, under this working condition, diesel oil can provide a more relaxed face contact pressure selection space to ensure the zero-leakage and long-life operation of the mechanical seal under the permissible wear rate. In addition, it can be seen that the lower limit $\left(p_{\mathrm{cmin}}\right)$ of the face contact pressure range of clean water, diesel oil and propane propylene is $0.477 \mathrm{MPa}$. Moreover, when the material of the rotating and stationary rings and the surface morphology parameters are determined, the minimum face contact pressure $\left(p_{\mathrm{cmin}}\right)$ corresponding to the zero-leakage condition of the sealing interface is found, and it has nothing to do with the sealing medium.

\subsection{The influence of the working condition parameters on the face contact pressure range}

The same calculation method is used to compute the face contact pressure, while changing the operating speed and fixing the other conditions. The calculation results are shown in Fig. 5. It can be observed that, as the speed increases, the face contact pressure ranges of the three-sealing mediums show a rapid shrinking trend. When the rotation speed reaches $4000 \mathrm{r} / \mathrm{min}$, the face contact pressure range of clean water and propane propylene becomes very narrow $(0.477 \sim 0.530$ MPa). In addition, under high-speed working conditions, the higher the lubrication performance of the sealing medium, the larger the obtained space of the face contact pressure range. This creates favorable conditions for the zero-leakage and long-life operation of the mechanical seal, under the allowable wear rate.

Fig. 6 shows the variation of the face contact pressure range function of the pressure of the sealing medium, while the other conditions are fixed. It can be seen that the increase in media pressure has the same influence on the face contact pressure range of the three-sealing media, which is embodied in the continuous reduction of the face contact pressure range when the medium pressure increases. By comparing Fig. 5 and Fig. 6, it can be deduced that the influence 
of the medium pressure on the face contact pressure range is lower than that of the operating speed.

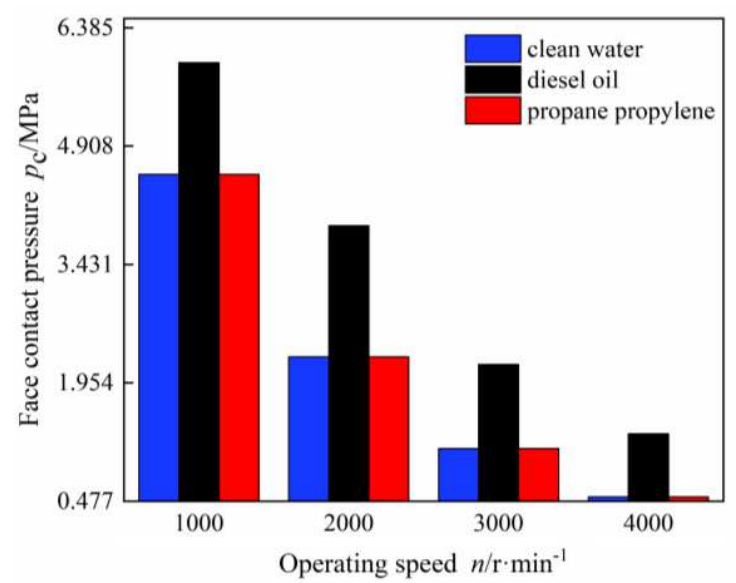

Fig. 5. Influence of the operating speed on the face contact pressure range

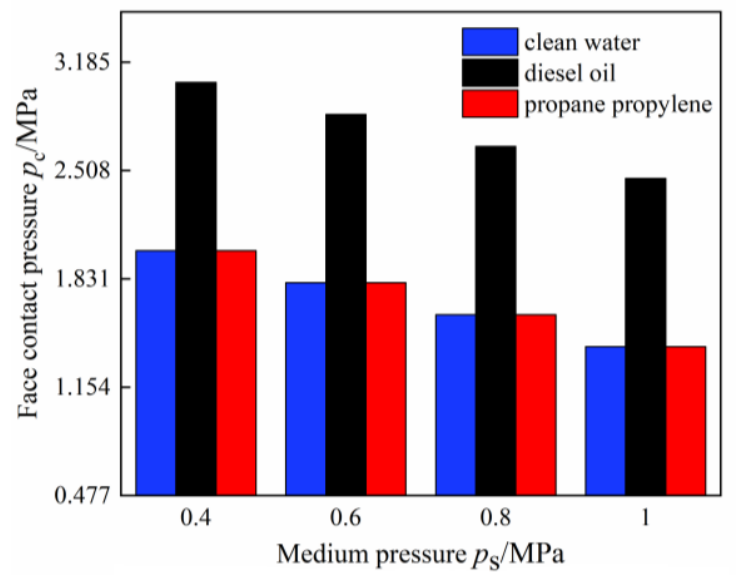

Fig. 6. Influence of the medium pressure on the face contact pressure range

Fig. 7 shows the influence of the medium temperature change on the face contact pressure range. It can be seen that, when the medium temperature varies between $35^{\circ} \mathrm{C}$ and $80^{\circ} \mathrm{C}$, the face contact pressure ranges of the clean water and diesel oil show a shrinking trend, while the reduction of diesel oil is more obvious. This is due to the fact that the lubrication performance of diesel oil is more susceptible to temperature than clean water, which results in increasing the wear coefficient of the sealing interface. In addition, the face contact pressure range of propane propylene is almost unchanged. Moreover, the influence of the medium temperature on the face contact pressure range is mainly affected by the change of the sealing medium lubrication performance. As the temperature increases, the lubrication environment between the seal 
interfaces deteriorates and the wear intensifies, which limits the face contact pressure range.

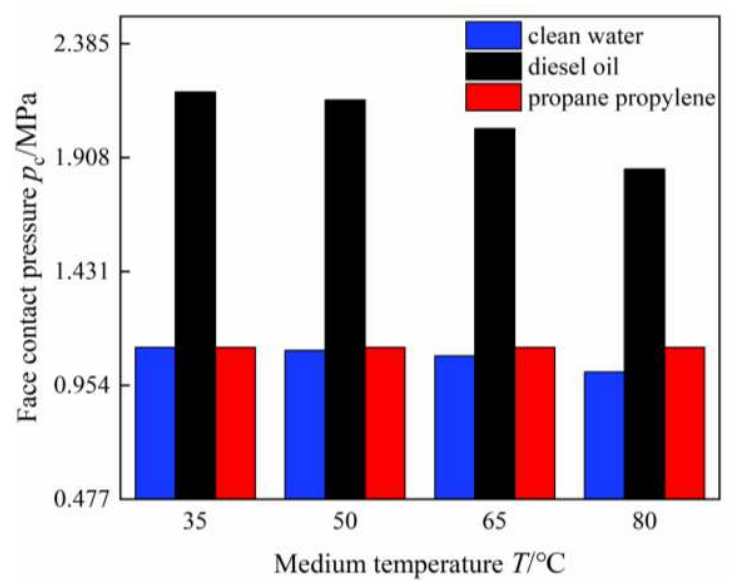

Fig. 7. Influence of the medium temperature on the face contact pressure range

\section{Conclusion}

(1) The critical porosity in the case of zero-leakage at the sealing interface, varies with the wettability of the sealed medium to the rotating and stationary rings materials. For the rotating and stationary rings that can be infiltrated by the sealing medium, there should be no microchannels at the sealing interface, with a critical porosity of 0.312 . On the contrary, for the rotating and stationary rings that cannot be infiltrated by the sealing medium, there may be microchannels on the sealing interface, and the critical porosity is that when the percolation point size is equal to the critical diameter of the microchannel.

(2) The wear rate is related to the temperature increase of the end face and the contact properties of the asperity. The asperity on the sealing interface is generally in elastic-plastic contact state. Its adhesive friction coefficient or wear rate can be characterized by the relationship between the friction coefficient or wear rate of elastic contact and plastic contact, as well as the template function.

(3) A design criterion of face contact pressure of zero-leakage mechanical seal is developed. The face contact pressure is well controlled, and the zero-leakage and long-life operation of the mechanical seal can be performed.

(4) This paper does not take into consideration the case where a leakage channel at the 
wettable seal interface exists while the zero-leakage of the mechanical seal can still be achieved, leaving this perspective an open discussion for future considerations.

\section{Author Contributions}

Zheng Wei: Term, Conceptualization, Methodology, Software, Writing - original draft. Sun Jianjun: Software, Supervision, Formal analysis. Ma Chenbo: Formal analysis. Yu Qiuping: Data curation.

\section{Funding}

This work was supported by the National Natural Science Foundation of China [grant number 52075268].

Data Availability

All data reported in a graphical format can be requested from the corresponding author.

\section{Code Availability}

Not applicable.

\section{Declarations}

\section{Conflicts of interest}

The authors declare that they have no known competing fnancial interests or personal relationships that could have appeared to infuence the work reported in this paper.

\section{Ethical Approval}

Not applicable.

\section{Consent to Participate}

We declare that this work was approved by all participants.

\section{Consent for Publication}

We hereby confrm that this manuscript is our original work and has not been published nor has it been submitted simultaneously elsewhere. We further confrm that all authors have checked the manuscript and have agreed to the submission.

\section{Nomenclature}




\begin{tabular}{|c|c|}
\hline$a_{\mathrm{ec}}$ & critical elastic deformation area, $\mathrm{m}^{2}$ \\
\hline$a_{\mathrm{pc}}$ & critical plastic deformation area, $\mathrm{m}^{2}$ \\
\hline$A_{\mathrm{a}}$ & nominal contact area of sealing surface, $\mathrm{m}^{2}$ \\
\hline$A_{\text {am }}$ & calculated nominal area, $\mathrm{m}^{2}$ \\
\hline$A_{\mathrm{r}}$ & actual contact area of sealing surface, $\mathrm{m}^{2}$ \\
\hline$b$ & half width of the sealing surface, $m$ \\
\hline$c_{\mathrm{p}}$ & specific heat capacity, $\mathrm{J} \cdot(\mathrm{kg} \cdot \mathrm{K})^{-1}$ \\
\hline$d$ & bottom diameter of the largest asperity, $\mu \mathrm{m}$ \\
\hline$d_{\max }$ & maximum contact diameter of the asperities, $\mu \mathrm{m}$ \\
\hline $\bar{d}$ & average diameter of the asperity, $\mu \mathrm{m}$ \\
\hline$\dot{d}$ & depth wear rate, $\mathrm{mm} \cdot 100 \mathrm{~h}^{-1}$ \\
\hline$D$ & fractal dimension of the sealing end surface \\
\hline$E^{*}$ & composite elastic modulus of the rotating and stationary rings, $\mathrm{MPa}$ \\
\hline$f$ & total friction coefficient of the sealing interface \\
\hline$f_{\mathrm{c}}$ & adhesive friction factor \\
\hline$f(a)$ & template function \\
\hline$G\left(p_{\mathrm{c}}\right)$ & characteristic function of face contact pressure and porosity \\
\hline$h$ & fluid film thickness in the non-contact zone of the sealing interface, $\mu \mathrm{m}$ \\
\hline$H_{\mathrm{s}}$ & $\begin{array}{l}\text { material hardness of the softer ring in the sealing interface matched } \\
\text { ring, } \mathrm{MPa}\end{array}$ \\
\hline$H\left(p_{\mathrm{c}}\right)$ & characteristic function of face contact pressure and wear rate \\
\hline$k$ & wear coefficient \\
\hline K & thermal conductivity, $\mathrm{W} \cdot(\mathrm{m} \cdot \mathrm{K})^{-1}$ \\
\hline$l$ & half width of the rectangular heat source in the $\mathrm{x}$ direction, $\mathrm{m}$ \\
\hline$L$ & Pelect number \\
\hline$n$ & rotating speed, $r \cdot \min ^{-1}$ \\
\hline$n(a)$ & the distribution function of contact spot area \\
\hline$n(a) a$ & number of asperities \\
\hline$p$ & capillary force, $\mathrm{N}$ \\
\hline$p_{\mathrm{c}}$ & face contact pressure, $\mathrm{MPa}$ \\
\hline$p_{\text {cmin }}$ & minimum face contact pressure, $\mathrm{MPa}$ \\
\hline$p_{\text {cmax }}$ & maximum face contact pressure, $\mathrm{MPa}$ \\
\hline$p_{\mathrm{g}}$ & specific load of the end face, MPa \\
\hline$p_{\mathrm{m}}$ & fluid film pressure, $\mathrm{MPa}$ \\
\hline$p_{\mathrm{s}}$ & medium pressure, $\mathrm{MPa}$ \\
\hline$q$ & heat flux density, $\mathrm{J} \cdot\left(\mathrm{m}^{2} \cdot \mathrm{s}\right)^{-1}$ \\
\hline$Q$ & frictional heat, $\mathrm{J}$ \\
\hline
\end{tabular}




\begin{tabular}{|c|c|}
\hline$\dot{Q}$ & thermal strength, $\mathrm{W}$ \\
\hline$Q / V$ & internal heat generation, $\mathrm{J} \cdot \mathrm{m}^{-3}$ \\
\hline$r$ & radius of the microchannel, $\mathrm{mm}$ \\
\hline$r_{\mathrm{c}}$ & critical radius of the zero-leakage microchannel, $\mathrm{mm}$ \\
\hline$r_{\mathrm{i}}$ & inner radius of the sealing surface, $\mathrm{mm}$ \\
\hline$r_{\mathrm{o}}$ & outer radius of the sealing surface, $\mathrm{mm}$ \\
\hline$S\left(p_{c}\right)$ & characteristic function of face contact pressure and microchannel radius \\
\hline$t$ & time, $\mathrm{s}$ \\
\hline$T$ & temperature rise, $\mathrm{K}$ \\
\hline$W$ & end face load, $\mathrm{N}$ \\
\hline$V$ & volume, $\mathrm{m}^{3}$ \\
\hline$U$ & linear velocity of the middle diameter of the sealing surface, $\mathrm{m} \cdot \mathrm{s}^{-1}$ \\
\hline$\alpha$ & component of the asperities contact area, $\mathrm{m}^{2}$ \\
\hline$\beta^{*}$ & composite correlation length of the asperity, $\beta^{*}=0.8, \mathrm{~mm}$ \\
\hline$\delta$ & compression of the asperity, $\mu \mathrm{m}$ \\
\hline$\zeta$ & surface tension of the liquid, $\mathrm{N}$ \\
\hline$\kappa$ & thermal diffusivity \\
\hline$\mu$ & fluid viscosity, $\mathrm{Pa} \cdot \mathrm{s}$ \\
\hline$\rho$ & density, $\mathrm{kg} \cdot \mathrm{m}^{-3}$ \\
\hline$\sigma$ & equivalent surface mean square error of asperities, $\mathrm{MPa}$ \\
\hline$\sigma_{1}$ & hard ring surface mean square error, $\mathrm{MPa}$ \\
\hline$\sigma_{2}$ & soft ring surface mean square deviation, $\mathrm{MPa}$ \\
\hline$\tau_{\mathrm{c}}$ & average value of the dry friction shear strength, $\mathrm{MPa}$ \\
\hline$\varphi$ & 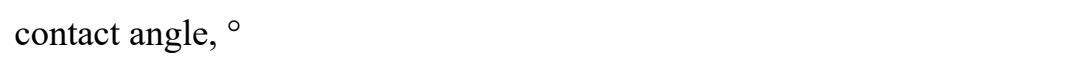 \\
\hline$\phi$ & porosity \\
\hline$\phi_{0}$ & initial porosity \\
\hline$\psi$ & area coefficient of the heat source, $\psi=1.12$ \\
\hline$\chi_{\mathrm{h}}$ & frictional heat component under high contact stress, $\mathrm{J}$ \\
\hline$\chi_{\mathrm{L}}$ & frictional heat component under low contact stress, $\mathrm{J}$ \\
\hline
\end{tabular}

\section{References}

[1] Hao M. Mechanical seal technology and application[M]. Beijing: China Petrochemical Press, 2010.

[2] Sun J. J., Ma C. B., Yu Q. P., et al. Numerical analysis on a new pump-out hydrodynamic mechanical seal[J]. Tribology International, 2017, 106: 62-70. 
[3] Du P. Y., Sheng J, Li J. Analysis of factors affecting the specific pressure of mechanical seals[J]. Journal of Beijing Institute of Petrochemical Technology, 2013, 21(4): 22-25.

[4] Sun J. J., Chen G. Q., Ji Z. B., et al. Analysis of interface leakage mechanism of contact mechanical seal[J]. CIESC Journal, 2018, 69(04): 282-290.

[5] Mayer E. Mechanical Seals[M]. London: Newnes- Butterworth, 1997.

[6] Lebeck A. O. Principles and design of mechanical face seals[M[. New York: A Wiley-Interscience Publication John Wiley \& Sons, Inc., 1991.

[7] Gu Y. Q. The selection principle of mechanical seal specific pressure[J]. Petrochemical Equipment, 2000, 29(2): 21-24.

[8] Gu Y. Q. Calculation of the main coefficients of mechanical end face seals (2)-Mechanical parameters and performance parameters[J]. Fluid Machinery, 1996, 24(5): 28-32.

[9] Salant R. F., Cao B. Unsteady analysis of a mechanical seal using Duhamel's method[C]. Proceedings of 2004 ASME/STLE international joint tribology conference, Long Beach, California USA, October 24-27, 2004.

[10] Elhanafi S., Farhang K. Leakage Prediction in Mechanical Seals Under Hydrostatic Operating Condition[C]. Proceedings of 2007 ASME/STLE international joint tribology conference, San Diego, California USA, October 22-24, 2007.

[11] Sun J. J., Gu B. Q., Wei L. Leakage model of contact mechanical seal based on fractal theory[J]. CIESC Journal, 2006, 57(7): 1626-1630.

[12] Li X. P., Yang Z. M., Wang L. L., et al. Leakage model of contact mechanical seal end face based on fractal theory[J]. Journal of Northeastern University (Natural Science Edition), 2019, 40(04): 73-77.

[13] Zhang P., Li S. X., Wang L., et al. Research on the end-face leakage rate of contact mechanical seals based on fluid-solid coupling[J]. Fluid Machinery, 2017(7): 17-21+87.

[14] Sun J. J., Ma C. B., Lu J. H., et al. A leakage channel model for sealing interface of mechanical face seals based on percolation theory[J]. Tribology International, 2018,118: 108-119.

[15] Ji Z. B. Research on interface leakage mechanism of contact mechanical seal based on percolation theory[D]. Nanjing Forestry University, 2018.

[16] Yuan T., Chen Z., Liu W. J., et al. Application of capillary mechanics to separation process 
of superhydrophilic membrane and its mechanical model[J]. Journal of South China University of Technology (NaturalScience Edition), 2014, 42(10 ): 82-89.

[17] Goilkar S. S., Hirani H. Parametric study on balance ratio of mechanical face seal in steam environment[J]. Tribology International, 2010, 43(5-6):1180-1185.

[18] Boylan, Joseph. Increasing seal-face capability in hard-on-hard combinations[J]. Sealing Technology, 2014, 2014(7):8-11.

[19] Sun J. J., Ji Z. B., Ma C. B. Reanalysis of the contact mechanics of rough surfaces[J]. Chinese Journal of Theoretical and Applied Mechani, 2018, 50(1): 68-77.

[20] Onions R. A., Archard J. F. The contact of surfaces having a random structure[J]. Journal of Physics D Applied Physics, 1973, 6(3): 289-304.

[21] ANSI/ASME B46.1. Surface Texture (Surface Roughness, Waviness, and Lay), New York: An American National Standards, ASME, 2009.

[22] Zhao Y. W., David D. M., Chang L. An asperity microcontact model incorporating the transition from elastic deformation to fully plastic flow[J]. ASME Journal of Tribology, 2000, 122: 86-93.

[23] Carslaw H. S., Jeager J. C. Conduction of heat in solids[M]. Oxford: Oxford University Press, 1986.

[24] Bhushan B. Ge S. R., trans. Introduction to Tribology[M]. Beijing: China Machinery Industry Press, 2006.

[25] Bhushan B. Magnetic head-media interface temperatures-part 1: analysis[J]. ASME J. Trib, 1987, 109: 243-251.

[26] Archard J. F. Contact and Rubbing of Flat Surfaces[J]. Journal of Applied Physics, 1953, 24(8): 981-988.

[27] Gu Y. Q. The budget of mechanical seal wear rate[J]. Petrochemical Equipment, 1999(01): 28-32.

[28] Lu J. H. Research on the leakage fluid flow characteristics between the contact mechanical seal interface[D]. Nanjing Forestry University, 2017.

[29] Ys A., Sza B., Jm C., et al. Failure Analysis of Graphite Stationary Ring Utilized in One Type of Mechanical Seal-ScienceDirect[J]. Engineering Failure Analysis, 2020, 108: 104-113.

[30] Gu Y. Q. Practical technology of mechanical seal[M]. Beijing: China Machinery Industry 
Press, 2001. 